\title{
Conservação de hepáticas na Mata Attântica do sudeste do Brasil: uma análise regional no estado do Rio de Janeiro ${ }^{1}$
}

\author{
Denise Pinheiro da Costa $^{2}$ e Nivea Dias dos $\operatorname{Santos}^{3}$
}

Recebido em 20/05/2008. Aceito em 29/10/2008

RESUMO - (Conservação de hepáticas na Mata Atlântica do sudeste do Brasil: uma análise regional no estado do Rio de Janeiro). A União Internacional para Conservação da Natureza (IUCN) desenvolveu um guia para avaliação do grau de ameaça das espécies em escala regional, que foi a base para a reavaliação do status de conservação das hepáticas no estado do Rio de Janeiro, após quatro anos da primeira análise. Dos 360 táxons ocorrentes no estado, 75 foram categorizados como ameaçados, sendo dois criticamente em perigo (CR), oito em perigo (EN) e 65 vulneráveis (VU). Além desses, quatro táxons foram categorizados como quase ameaçados (NT) e 12 com dados deficientes (DD). O elevado número de táxons ameaçados no estado (21\% da flora) reflete a importância do grau de conhecimento da flora para realização de análises de conservação. Em relação à distribuição dos táxons ameaçados dentro do estado, percebe-se que a maioria ocorre no PARNA Itatiaia (51 espécies - 68\%). As principais ameaças para a flora são a perda e degradação da Mata Atlântica. Recomenda-se então, reconhecimento da Mata Atlântica como área crítica para conservação; a proteção de habitats para assegurar a sobrevivência das espécies; e a formulação de programas multidisciplinares para promover a conservação.

Palavras-chave: briófitas, IUCN, Mata Atlântica, Rio de Janeiro, status de conservação

\begin{abstract}
Liverwort conservation in the Atlantic Rain Forest of Southeastern Brazil: a regional survey in Rio de Janeiro state). The International Union for the Conservation of Nature and Natural Resources (IUCN) developed a guide for assessment of species' threat level at a regional scale, which was the basis for the reassessment of liverwort conservation status in the state of Rio de Janeiro, four years after the first analysis. Of 360 taxa occurring in the state, 75 were categorized as threatened, two species were categorized as critically endangered (CR), eight endangered (EN) and 65 vulnerable (VU). Besides these, four species were categorized as almost threatened (NT), and 12 with poor data (DD). The high number of threatened taxa in the state (21\% of the flora) reflects the importance of the level of knowledge for flora conservation analysis. In relation to the distribution of threatened taxa within the state, the majority occur in Itatiaia National Park (51 species - 68\%). The main threats to the flora are loss and degradation of the Atlantic Rainforest. We recommend the recognition of the Atlantic Rainforest as a critical area for conservation; the protection of habitats to ensure the survival of species; and formulation of multidisciplinary programs to promote conservation.
\end{abstract}

Key words: Atlantic Rainforest, bryophytes, conservation status, IUCN, Rio de Janeiro

\section{Introdução}

A Mata Atlântica estende-se do Rio Grande do Norte ao Rio Grande do Sul, incluindo partes da Argentina e Paraguai (Silva \& Casteleti 2005), ocorrendo em regiões costeiras e do interior sobre montanhas e platôs.

Este importante ecossistema brasileiro apresenta elevada biodiversidade, devido às variações de relevo, regimes pluviométricos e unidades fitogeográficas (Rambaldi et al. 2003; Pinto et al. 2006). Estima-se que a região abrigue de 1 a $8 \%$ da biodiversidade mundial, com 20.000 espécies de plantas, sendo 40\% endêmicas (Myers et al. 2000; Mittermeier et al. 2004). Contudo, vem sofrendo um processo intensivo de destruição pelo corte ilegal, cultivo e ocupação humana, onde apenas $7-8 \%$ da área original (ca. 1,5 milhão de $\mathrm{km}^{2}$ ) persistem como um mosaico de fragmentos isolados, o que torna a Mata Atlântica uma das regiões mais devastadas e seriamente ameaçadas do planeta (Galindo-Leal \& Câmara 2005), sendo apontada por Gradstein \& Raeymaekers (2000) como uma das cinco áreas críticas para a conservação no Neotrópico.

O estado do Rio de Janeiro possui uma área de 43.864,3 $\mathrm{km}^{2}$ de extensão, compreendendo 91 municípios (Fundação CIDE 2006). Por sua localização e diversidade de formações geográficas, é caracterizado por uma alta diversidade bio- lógica, relacionada à grande variedade de habitats (campos de altitude, restingas com formações arbóreas e arbustivas, lagoas ao longo das regiões costeiras, mangues, e florestas de terras baixas, submontana, montana e alto-montana), assim como pelo alto endemismo, demonstrando a importância de sua flora e fauna (Projeto Flora do Estado do Rio de Janeiro 2002).

Estima-se que antes da descoberta do Brasil, 97\% do estado do Rio de Janeiro era recoberto pela Mata Atlântica. Os 500 anos de destruição da floresta causaram a perda de grande parte desse importante ecossistema, estando a cobertura remanescente atualmente reduzida a ca. $19 \%$ da vegetação do estado (Fundação S.O.S. Mata Atlântica 2002).

Por muitos anos as briófitas foram negligenciadas em programas de conservação, principalmente pela falta de conhecimento da distribuição e ecologia desse grupo de plantas. Atualmente, diversos países europeus possuem checklists e listas vermelhas; e em 1999, a IUCN (União Internacional para Conservação da Natureza) através do grupo de especialistas em briófitas (Species Survival Commission) adaptou as regras da IUCN, criando um Plano de Ação para a conservação das espécies de briófitas no mundo (Hallingbäck \& Hodgetts 2000). Nesse plano, 92 espécies são citadas com diferentes graus de ameaça, incluindo duas espécies do Brasil categorizadas como em perigo de extinção

\footnotetext{
1 Parte da Dissertação de Mestrado da segunda Autora no Programa de Pós-graduação em Botânica da Escola Nacional de Botânica Tropical/ Instituto de Pesquisas Jardim Botânico do Rio de Janeiro

2 Instituto de Pesquisas Jardim Botânico do Rio de Janeiro, Programa Diversidade Taxonômica, Rua Pacheco Leão 915, 22460-030 Rio de Janeiro, RJ, Brasil

3 Departamento de Botânica, Instituto de Biologia, Universidade Estadual de Campinas, 13083-970 Campinas, SP, Brasil

2 Autor para contato: dcosta@jbrj.gov.br
} 
(EN), Drepanolejeunea aculeata Bischl. e Myriocoleopsis fluviatilis (Steph.) E. Reiner \& Gradst.

No Brasil, os primeiros trabalhos que tratam de conservação de briófitas começaram a ser realizados a partir de 1999, incluindo análises nacionais e regionais. Análises a nível nacional foram realizadas por Costa (dados não publicados), na revisão da família Metzgeriaceae para o Brasil, onde seis espécies de Metzgeria foram caracterizadas como vulneráveis no país; e por Yano et al. (2005), que elaboraram a primeira lista vermelha de briófitas para o Brasil, onde estão citadas 17 espécies, recentemente reconhecidas oficialmente pelo Ministério do Meio Ambiente na Instrução Normativa $\mathrm{n}^{\circ} 6$ de 23 de setembro de 2008 como ameaçadas.

Em nível regional, existem quatro importantes trabalhos que tratam das espécies de briófitas da Mata Atlântica em diferentes regiões do país: Pôrto \& Germano (2002), que analisaram a brioflora do estado de Pernambuco, considerando 20 táxons vulneráveis (6,5\%); Costa et al. (2005a), analisando a brioflora do estado do Rio de Janeiro, caracterizando 150 espécies com algum grau de ameaça (14\%); Costa et al. (2006a) que elaboraram a lista vermelha de briófitas do estado de Minas Gerais, onde estão incluídas 26 espécies $(2,8 \%)$ e cujas informações encontram-se disponíveis atualmente on-line (http://www.biodiversitas.org.br/listas-mg); e finalmente, Yano \& Peralta (2007), que analisaram a brioflora do estado do Espírito Santo, considerando 36 táxons (9\%) ameaçados para o estado.

Seguindo as recomendações da IUCN $(2001,2003)$, o principal objetivo desse trabalho é o de reavaliar o status de conservação dos táxons de hepáticas da Mata Atlântica do estado do Rio de Janeiro, após quatro anos da primeira análise realizada por Costa et al. (2005a), identificando o grau de ameaça da flora de hepáticas nesse importante ecossistema e fornecendo subsídios para a política de conservação no estado.

Histórico dos estudos sobre a flora de hepáticas do estado do Rio de Janeiro

A flora de hepáticas do Rio de Janeiro é considerada bem conhecida quando comparada à de outros estados brasileiros, sendo a segunda em termos de diversidade de espécies no país (Gradstein \& Costa 2003).

O conhecimento sobre a diversidade de hepáticas no estado inicia-se no princípio do século XIX, com a chegada de naturalistas viajantes no Brasil. Trabalhos clássicos como os de Bescherelle (1893), Dusén (1903), Hooker \& Wilson (1844), Massalongo (1911), Montagne (1839), Nees (1833), Raddi (1822), Spruce (1888), Stephani (1897), entre outros, baseados nas coleções de Auguste F.M. Glaziou, Auguste de Saint-Hilaire, Ernest Ule, George Gardner, Carl F.V. von Martius, Anders F. Regnell, Giuseppe Raddi e Mons. V. Mazzucchelli, são de suma importância para o conhecimento da flora de hepáticas do estado.

A partir da década de 1980, diversos trabalhos vêm sendo desenvolvidos, representando valiosas contribui- ções para o conhecimento da flora de hepáticas do estado, destacando-se os trabalhos de Costa (1992; 1997; 1999), Costa (2008), Costa \& Yano (1988, 1998), Costa et al. (2006b), Molinaro \& Costa (2001), Oliveira-e-Silva \& Yano (2000), Oliveira-e-Silva et al. (2002), Santos \& Costa (2008) e Yano \& Costa (1998).

Os catálogos de Yano (1984, 1989, 1995, 1996 e 2006), sumarizam a literatura sobre briófitas no Brasil e a informação contida neles constitui uma fonte essencial para estudos briológicos no país.

A primeira flora de hepáticas do Brasil foi realizada por Gradstein \& Costa (2003) onde estão citadas 367 espécies de hepáticas para o estado do Rio de Janeiro. Posteriormente, Costa et al. (2005a,b) elaboraram o primeiro checklist de briófitas para o estado, onde citam 333 espécies de hepáticas, e a primeira análise da diversidade e importância das briófitas para a conservação dos ecossistemas do Rio de Janeiro, onde citam 68 espécies de hepáticas com algum grau de ameaça no estado (11 em perigo e 57 vulneráveis).

\section{Material e métodos}

Para a reavaliação do status de conservação dos táxons de hepáticas categorizados por Costa et al. (2005a) com algum grau de ameaça no estado, este estudo associou dados de campo, informações do banco de dados da brioflora do estado do Rio de Janeiro (Costa et al. 2007) e dados da coleção de briófitas do herbário RB.

Foram realizadas 11 excursões, tendo sido coletados um total de 736 espécimes, em seis unidades de conservação do estado, a saber: APA Petrópolis (município de Petrópolis), PARNA Itatiaia (município de Itatiaia), PARNA Serra dos Órgãos (município de Teresópolis), PARNA Tijuca (município do Rio de Janeiro); PE Desengano (municípios de Santa Maria Madalena, São Fidélis e Campos); e RPPN El Nagual (município de Magé). Além disso, foram examinadas 120 exsicatas depositadas no herbário RB, pertencentes ao município de Nova Friburgo.

A análise regional do status de conservação dos táxons de hepáticas no estado do Rio de Janeiro se baseou no Plano de Ação para a conservação de espécies de briófitas da IUCN (Hallingbäck \& Hodgetts 2000), e as informações utilizadas se referem principalmente à distribuição dos táxons, número de localidades onde ocorrem e perda do habitat nos últimos anos, três dos cinco itens citados por Hallingbäck et al. (1998), no Guia para Aplicação das Categorias de Ameaças para Briófitas da IUCN, como os mais importantes para avaliação do grupo.

Na distribuição dos táxons no Brasil, a seqüência dos estados é apresentada por região geográfica e os nomes estão abreviados de acordo com o IBGE, como apresentados a seguir: Região Norte (RR - Roraima, RO - Rondônia, AP - Amapá, AC - Acre, AM - Amazonas, PA - Pará, TO Tocantins); Região Nordeste (MA - Maranhão, PI - Piauí, CE - Ceará, RN - Rio Grande do Norte, PB - Paraíba, PE - Pernambuco, AL - Alagoas, $\mathrm{SE}$ - Sergipe, BA - Bahia); Região Centro-oeste (GO - Goiás, MT - Mato Grosso, MS - Mato Grosso do Sul); Região Sudeste (MG - Minas Gerais, ES - Espírito Santo, RJ - Rio de Janeiro, SP - São Paulo); Região Sul (PR - Paraná, SC - Santa Catarina, RS - Rio Grande do Sul).

\section{Resultados e discussão}

Dos 360 táxons de hepáticas reconhecidos por Santos \& Costa (no prelo) para o estado do Rio de Janeiro, 68 foram considerados por Costa et al. (2005a) com algum grau de ameaça no estado, sendo 57 vulneráveis (VU) e 11 em perigo (EN). Dessas 68 espécies, 38 (56\%) foram coletadas nas áreas estudadas (Tab. 1). Das 14 espécies incluídas em dados deficientes (DD) por Costa et al. (2005a), duas foram 
Tabela 1. Lista das espécies de hepáticas reavaliadas quanto ao seu status de conservação no Estado do Rio de Janeiro, Brasil, com base na lista vermelha de Costa et al. $(2005 \mathrm{a}) . *$ coletada recentemente. Negrito $=$ espécies que mudaram de categoria. Categorias IUCN: $\mathrm{CR}=\mathrm{criticamente}$ em perigo, EN = em perigo, VU = vulnerável, NT = quase ameaçada, LC = não ameaçada.

\begin{tabular}{|c|c|c|c|c|c|}
\hline \multirow[b]{2}{*}{ Táxon } & \multirow[b]{2}{*}{$\begin{array}{l}\text { Localidade de ocorrência no } \\
\text { estado }\end{array}$} & \multirow[b]{2}{*}{$\begin{array}{l}\text { Altitude no } \\
\text { Brasil (m) }\end{array}$} & \multirow[b]{2}{*}{$\begin{array}{l}\text { Distribuição geográfica } \\
\text { no mundo e Brasil }\end{array}$} & \multicolumn{2}{|c|}{ Categoria IUCN } \\
\hline & & & & $\begin{array}{l}\text { Costa et al. } \\
\text { (2005a) }\end{array}$ & $\begin{array}{c}\text { Este } \\
\text { trabalho }\end{array}$ \\
\hline \multicolumn{6}{|l|}{ TÁXON NÃO AMEAÇADO (1) } \\
\hline *Plagiochila patula (Sw.) Lindenb. & $\begin{array}{l}\text { REBIO Tinguá, Itatiaia, Serra } \\
\text { dos Órgãos, RPPN E1 Nagual, PE } \\
\text { Desengano }\end{array}$ & $100-2100$ & Neotropical; RJ & $\mathrm{VU}$ & $\mathrm{LC}$ \\
\hline \multicolumn{6}{|l|}{ TÁXONS QUASE AMEAÇADOS (4) } \\
\hline *Calypogeia uncinulatula Herzog & Itatiaia e Serra dos Órgãos & $800-1200$ & $\begin{array}{l}\text { Neotropical; GO, MG, } \\
\text { RJ, SP }\end{array}$ & VU & NT \\
\hline $\begin{array}{l}\text { *Cryptochila grandiflora (Lindenb. \& Gottsche) } \\
\text { Grolle }\end{array}$ & Itatiaia, Friburgo, Serra dos Órgãos & $1750-2500$ & Ampla; ES, RJ & VU & NT \\
\hline *Heteroscyphus combinatus (Nees) Schiffn. & $\begin{array}{l}\text { Serra dos Órgãos, Nova Friburgo, } \\
\text { RPPN El Nagual, PE Desengano }\end{array}$ & $0-1200$ & $\begin{array}{l}\text { Neotropical; AM, MG, } \\
\text { RJ, SP }\end{array}$ & VU & NT \\
\hline *Lophocolea mandonii Steph. & $\begin{array}{l}\text { Itatiaia, PARNA Tijuca, RPPN El } \\
\text { Nagual }\end{array}$ & $200-2500$ & Bolívia e Brasil; MG, RJ & VU & NT \\
\hline \multicolumn{6}{|l|}{ TÁXONS VULNERÁVEIS (52) } \\
\hline Anastrophyllum auritum (Lehm.) Steph. & Itatiaia & $1300-1500$ & Ampla; MG, RJ & VU & $\mathrm{VU}$ \\
\hline *Anastrophyllum piligerum (Nees) Steph. & Itatiaia e Serra dos Órgãos & $500-1550$ & Pantropical; MG, RJ, SP & $\mathrm{VU}$ & VU \\
\hline Aphanolejeunea asperrima Steph. & Itatiaia & $1450-2000$ & $\begin{array}{l}\text { Patagônia e Brasil; MG, } \\
\text { RJ, SC. }\end{array}$ & VU & VU \\
\hline *Aphanolejeunea paucifolia (Spruce) E. Reiner & Arboreto JBRJ e RPPN El Nagual & $0-1350$ & $\begin{array}{l}\text { Sul da América do Sul; } \\
\text { BA, MG, ES, RJ }\end{array}$ & $\mathrm{VU}$ & $\mathrm{VU}$ \\
\hline *Aphanolejeunea sintenisii (Steph.) Steph. & Itatiaia & $1100-1900$ & Porto Rico e Brasil; RJ. & VU & VU \\
\hline *Aureolejeunea fulva R.M. Schust. & Itatiaia & $2200-2600$ & Neotropical; MG, RJ & VU & $\mathrm{VU}$ \\
\hline $\begin{array}{l}\text { *Bazzania cuneistipula (Gottsche \& Lindenb.) } \\
\text { Trevis. }\end{array}$ & Itatiaia & $1200-1400$ & $\begin{array}{l}\text { Norte dos Andes, Antilhas } \\
\text { e Brasil; RJ, SP }\end{array}$ & $\mathrm{VU}$ & $\mathrm{VU}$ \\
\hline Bazzania schlimiana (Gottsche) Fulford. & Itatiaia & $800-2000$ & Neotropical; RJ, SP. & VU & VU \\
\hline *Bazzania taleana (Gottsche) Fulford. & Itatiaia e Serra dos Órgãos & $1200-2100$ & Neotropical; RJ, SP, SC & VU & VU \\
\hline $\begin{array}{l}\text { *Blepharolejeunea incongrua (Lindenb. \& } \\
\text { Gottsche) Van Slageren \& Kruijt }\end{array}$ & Itatiaia & $1650-2600$ & Neotropical; MG, RJ & VU & VU \\
\hline $\begin{array}{l}\text { Blepharolejeunea securifolia (Steph.) R.M. } \\
\text { Schust. }\end{array}$ & Itatiaia & $>2000$ & Neotropical; RJ & VU & VU \\
\hline *Calypogeia grandistipula (Steph.) Steph. & Itatiaia & $800-2000$ & Brasil; MG, ES, RJ, SP & VU & VU \\
\hline *Cephalozia crossi Spruce & Itatiaia & $1700-2300$ & Neotrópical; ES, MG, RJ & $\mathrm{VU}$ & VU \\
\hline *Cephaloziella granatensis (J.B. Jack) Fulford. & Serra dos Órgãos & $1000-2000$ & $\begin{array}{l}\text { Neotrópico e Madeira; } \\
\text { MG, RJ }\end{array}$ & $\mathrm{VU}$ & VU \\
\hline $\begin{array}{l}\text { Cephaloziopsis intertexta (Gottsche) R.M. } \\
\text { Schust. }\end{array}$ & REBIO Tinguá e Serra dos Órgãos & $0-1400$ & Neotropical; RJ, SP & $\mathrm{VU}$ & VU \\
\hline *Colura calyptrifolia (Hook.) Dumort. & Itatiaia & $2350-2400$ & Ampla; MG, RJ & VU & VU \\
\hline Colura itatyana Steph. & Itatiaia & $2300-2400$ & Brasil; RJ & VU & VU \\
\hline Diplasiolejeunea pauckertii (Nees) Steph. & Itatiaia & $1500-2300$ & $\begin{array}{l}\text { Norte dos Andes e Brasil; } \\
\text { MG, RJ }\end{array}$ & VU & $\mathrm{VU}$ \\
\hline $\begin{array}{l}\text { *Diplasiolejeunea unidentata (Lehm. \& } \\
\text { Lindenb.) Schiffn. }\end{array}$ & $\begin{array}{l}\text { Serra dos Órgãos e REBIO Poço } \\
\text { das Antas }\end{array}$ & $0-1000$ & $\begin{array}{l}\text { Neotropical; PE, BA, RJ, } \\
\text { SP, SC }\end{array}$ & VU & VU \\
\hline $\begin{array}{l}\text { *Drepanolejeunea granatensis (J.B. Jack \& } \\
\text { Steph.) Bischl. }\end{array}$ & Itatiaia & $>2000$ & $\begin{array}{l}\text { Andes e SE do Brasil: MG, } \\
\text { RJ, SP }\end{array}$ & VU & $\mathrm{VU}$ \\
\hline *Frullania ecklonii (Spreng.) Gottsche et al. & Itatiaia e Serra dos Órgãos & $0-2400$ & $\begin{array}{l}\text { Pantropical; AC, GO, } \\
\text { MG, RJ }\end{array}$ & $\mathrm{VU}$ & VU \\
\hline $\begin{array}{l}\text { Frullania intumescens (Lehm. \& Lindenb.) } \\
\text { Lehm. \& Lindenb. }\end{array}$ & sem localidade & $500-1000$ & Neotropical; MG, RJ & VU & $\mathrm{VU}$ \\
\hline Frullania schäfer-verwimpii Yuzawa \& Hatt. & Serra dos Órgãos & $0-1100$ & Brasil; MG, RJ, SP & $\mathrm{VU}$ & $\mathrm{VU}$ \\
\hline $\begin{array}{l}\text { *Gongylanthus liebmanianus (Lindenb. \& } \\
\text { Gottsche) Steph. }\end{array}$ & Itatiaia e Serra dos Órgãos & $>2000$ & Neotropical; ES, RJ & VU & VU \\
\hline *Harpalejeunea subacuta A. Evans & Itatiaia & $2000-2400$ & $\begin{array}{l}\text { Antilhas e Brasil; MG, } \\
\text { RJ, SP }\end{array}$ & VU & VU \\
\hline Herbertus grossispinus (Steph.) Fulford & Serra dos Órgãos e APA Petrópolis & ca. 2000 & Neotropical; ES, RJ & VU & VU \\
\hline Herbertus oblongifolius (Steph.) Gradst. \& Cleef & Itatiaia & ca. 1800 & Colômbia e Brasil; RJ & $\mathrm{VU}$ & $\mathrm{VU}$ \\
\hline *Jungermannia hyalina Lyell & Itatiaia e Serra dos Órgãos & $500-2400$ & Holártica; MT, MS, MG, RJ & $\mathrm{VU}$ & VU \\
\hline Jungermannia sphaerocarpa Hook. & Itatiaia & $2000-2500$ & Holártica; RJ & VU & $\mathrm{VU}$ \\
\hline Kurzia flagellifera (Steph.) Grolle & Itatiaia & ca. 1000 & Neotropical; GO RJ, SP & VU & VU \\
\hline Leptoscyphus gibbosus (Tayl.) Mitt. & Itatiaia & ca. 800 & $\begin{array}{l}\text { Antilhas, Venezuela e } \\
\text { Brasil; RJ. }\end{array}$ & VU & $\mathrm{VU}$ \\
\hline
\end{tabular}


Tabela 1 (Continuação).

\begin{tabular}{|c|c|c|c|c|c|}
\hline \multirow[b]{2}{*}{ Táxon } & \multirow[b]{2}{*}{$\begin{array}{l}\text { Localidade de ocorrência no } \\
\text { estado }\end{array}$} & \multirow[b]{2}{*}{$\begin{array}{l}\text { Altitude } \\
\text { no Brasil } \\
\text { (m) }\end{array}$} & \multirow[b]{2}{*}{$\begin{array}{l}\text { Distribuição geográfica } \\
\text { no mundo e Brasil }\end{array}$} & \multicolumn{2}{|c|}{ Categoria IUCN } \\
\hline & & & & $\begin{array}{l}\text { Costa et al. } \\
\text { (2005a) }\end{array}$ & $\begin{array}{c}\text { Este } \\
\text { trabalho }\end{array}$ \\
\hline Lophocolea connata (Sw.) Nees & Serra dos Órgãos & $500-2000$ & Brasil; RJ & VU & VU \\
\hline *Lophozia bicrenata (Schmid.) Dumort. & Itatiaia & $2200-2400$ & Holártica; ES, RJ & VU & VU \\
\hline Marchantia breviloba A. Evans & Itatiaia e Parati & $900-1350$ & Neotropical; RJ & VU & VU \\
\hline *Marsupella microphylla R.M. Schust. & Itatiaia & $2300-2400$ & Venezuela e Brasil; MG, RJ & VU & VU \\
\hline Mnioloma cyclostipa (Spruce) R.M. Schust. & Itatiaia & $1300-1900$ & $\begin{array}{l}\text { América Central, Guiana, } \\
\text { Andes (tropicais) e Sudeste } \\
\text { do Brasil; MG, RJ }\end{array}$ & VU & VU \\
\hline Neesioscyphus carneus (Nees) Grolle & Rio de Janeiro e Serra dos Órgãos & $500-1400$ & Brasil; MG, ES, RJ, SP, SC & VU & VU \\
\hline *Neesioscyphus homophyllus (Nees) Grolle & Serra da Bocaina & $500-1500$ & Brasil; MG, RJ, SP & VU & VU \\
\hline Odontolejeunea decemdentata (Spruce) Steph. & Itatiaia & $0-1150$ & Neotropical; RJ, SP & VU & VU \\
\hline *Paracromastigum dusenii (Steph.) R.M. Schust. & Itatiaia & $2350-2400$ & Chile temperado e Brasil; RJ & VU & VU \\
\hline *Plagiochila diversifolia Lindenb. \& Gottsche & Serra dos Órgãos e Friburgo & $1300-2000$ & Neotropical; BA, MG, RJ & VU & VU \\
\hline * Plagiochila exigua (Tayl.) Tayl. & Itatiaia & $1500-2400$ & Ampla; MG, RJ, SP & VU & VU \\
\hline * Plagiochila macrostachya Lindenb. & Itatiaia e Serra dos Órgãos & $1500-2200$ & Neotropical; MG, RJ, SP & VU & VU \\
\hline *Radula fendleri Gottsche & Itatiaia & $1000-1700$ & $\begin{array}{l}\text { Antilhas e América do Sul } \\
\text { tropical; MG, ES, RJ, SP }\end{array}$ & VU & VU \\
\hline Radula gottscheana Tayl. & Itatiaia & $0-1200$ & Neotropical; AM, RJ & VU & VU \\
\hline Radula pocsii $\mathrm{K}$. Yamada & Itatiaia & ca. 1450 & Cuba e Brasil; RJ & VU & VU \\
\hline *Radula schäfer-verwimpii K. Yamada & Itatiaia & $900-2300$ & $\begin{array}{l}\text { Colômbia e Brasil; MG, } \\
\text { ES, RJ, SP }\end{array}$ & VU & VU \\
\hline *Stephaniella paraphyllina J.B. Jack & Itatiaia & $2100-2500$ & Afro-americana; MG, RJ & $\mathrm{VU}$ & VU \\
\hline *Syzygiella integerrima Steph. & Itatiaia e Serra dos Órgãos & $1700-2300$ & Neotropical; MG, ES, RJ & VU & VU \\
\hline *Syzygiella liberata Inoue & Itatiaia e Serra dos Órgãos & $1750-2280$ & $\begin{array}{l}\text { Costa Rica, Andes, Brasil; } \\
\text { MG, RJ, SC }\end{array}$ & VU & VU \\
\hline Syzygiella uleana Steph. & Itatiaia e Friburgo & $1400-2000$ & Brasil; MG, RJ & VU & VU \\
\hline $\begin{array}{l}\text { *Triandrophyllum subtrifidum (Hook.f. \& Tayl.) } \\
\text { Fulford \& Hatch. }\end{array}$ & Itatiaia & $2000-2500$ & $\begin{array}{l}\text { Regiões temperadas do } \\
\text { hemisfério Sul, montanhas } \\
\text { altas da América e Ásia } \\
\text { tropicais; MG, ES, RJ }\end{array}$ & $\mathrm{VU}$ & VU \\
\hline \multicolumn{6}{|l|}{ TÁXONS EM PERIGO (11) } \\
\hline Bromeliophila natans (Steph.) R.M. Schust. & Praia de Mauá, Magé & nível do mar & Brasil; RJ, SP & EN & $\mathrm{CR}$ \\
\hline Calypogeia lechleri (Steph.) Steph. & Pão de Açúcar & $0-800$ & Neotropical; RJ, SP, RS & $\mathrm{EN}$ & EN \\
\hline Cheilolejeunea inflexa (Hampe) Grolle & Itatiaia e Rio de Janeiro & $500-2600$ & Neotropical; RJ & EN & EN \\
\hline Drepanolejeunea aculeata Bischl. & Rio de Janeiro & $0-1000$ & Brasil; RJ, SP & EN & EN \\
\hline Frullania gaudichaudii (Nees \& Mont.) Nees \& Mont. & Rio de Janeiro & $0-200$ & Guiana e Brasil; MT, RJ & EN & EN \\
\hline *Jensenia spinosa (Lindenb. \& Gottsche) Grolle & Itatiaia e Serra dos Órgãos & $750-2400$ & Afroamericana; RJ & $\mathrm{EN}$ & VU \\
\hline *Lejeunea grossiretis (Steph.) E. Reiner \& Goda & $\begin{array}{l}\text { Petrópolis, PARNA Tijuca e PE } \\
\text { Desengano }\end{array}$ & $50-700$ & Brasil; BA, RJ & EN & VU \\
\hline Microlejeunea subulistipa Steph. & Itatiaia & $0-2000$ & Brasil; RJ, SP, SC & EN & EN \\
\hline *Odontoschisma brasiliense Steph. & Rio de Janeiro & $0-500$ & $\begin{array}{l}\text { Norte da América do Sul, } \\
\text { Brasil; AM, MG, RJ }\end{array}$ & $\mathrm{EN}$ & EN \\
\hline Plagiochila boryana Gottsche & Petrópolis & 1900 & Afro-americana; RJ & EN & EN \\
\hline Southbya organensis Herzog & Petrópolis & $1900-2200$ & Brasil, RJ & $\mathrm{EN}$ & $\mathrm{CR}$ \\
\hline
\end{tabular}

encontradas em diferentes localidades, formações e unidades de conservação (UCs), sendo aqui categorizadas como não ameaçadas (LC). São elas: Aneura pinguis (L.) Dumort., presente no PARNA Itatiaia, PARNA Tijuca, PE Desengano e RPPN El Nagual; e Riccardia emarginata (Steph.) Hell, coletada no município de Nova Friburgo, PARNA Tijuca, PE Desengano e RPPN El Nagual.

A reavaliação do status de conservação dos táxons de hepáticas no estado evidenciou que, dos 68 táxons considerados por Costa et al. (2005a) como vulneráveis (VU) ou em perigo (EN), nove (13\%) mudaram de categoria (Tab. 1). Além disso, doze táxons categorizados como de baixo risco (LC) por esses autores, são aqui indicados como ameaçados no estado por um ou mais dos motivos apresentados a seguir: área de ocupação restrita; ocorrência em poucas localidades; última coleção realizada há mais de 100 anos (Tab. 2). Desses táxons, um foi incluído na categoria em perigo (EN) e 11 na categoria de vulnerável (VU).

Dos 57 táxons caracterizados anteriormente como vulneráveis (VU), cinco tiveram seu grau de ameaça reduzido, com quatro deles passando para a categoria de quase ameaçados (NT) e um, Plagiochila patula (Sw.) Lindenb., para a categoria de não ameaçado (LC). Cinqüenta e três táxons permaneceram como vulneráveis (VU).

Dos onze táxons anteriormente categorizados como em perigo (EN), dois tiveram seu grau de ameaça aumentado, passando para a categoria de criticamente em perigo (CR), e dois tiveram seu grau de ameaça reduzido, passando para 
Tabela 2. Espécies de hepáticas aqui indicadas como ameaçadas no estado do Rio de Janeiro.

\begin{tabular}{|c|c|c|}
\hline Espécie & $\begin{array}{l}\text { Categoria } \\
\text { IUCN }\end{array}$ & Informações que basearam a mudança de categoria \\
\hline Isotachis inflata Steph. & EN & $\begin{array}{l}\text { Endêmica do estado do Rio de Janeiro (Fulford 1963; Hatcher 1961), conhecida por coleções antigas de Glaziou } \\
\text { (Rio de Janeiro) e de Dusén e Ule (Itatiaia), não tendo sido recoletada há mais de } 100 \text { anos. }\end{array}$ \\
\hline $\begin{array}{l}\text { Anomoclada portoricensis (Hampe \& } \\
\text { Gottsche) Váña }\end{array}$ & VU & $\begin{array}{l}\text { Espécie de distribuição neotropical, conhecida no Brasil para os estados de RR, AM e RJ, recordada no estado } \\
\text { por apenas uma coleção, realizada por Ule em Petrópolis (Fulford 1968), há mais de } 100 \text { anos. }\end{array}$ \\
\hline $\begin{array}{l}\text { Cylindrocolea planifolia (Steph.) } \\
\text { R.M. Schust. }\end{array}$ & VU & $\begin{array}{l}\text { Espécie neotropical, com ampla distribuição no Brasil (RO, AC, BA, MG, RJ, SP, SC). Conhecida para o estado } \\
\text { do Rio de Janeiro apenas por duas coleções, uma realizada na cidade do Rio de Janeiro, por Mosén, e outra em } \\
\text { Itatiaia, por Puiggari (Fulford 1976), não tendo sido recoletada há mais de } 100 \text { anos. }\end{array}$ \\
\hline $\begin{array}{l}\text { Cheilolejeunea insecta Grolle \& } \\
\text { Gradst. }\end{array}$ & VU & $\begin{array}{l}\text { Espécie disjunta entre a Serra da Mantiqueira (MG, RJ, SP) e o sul da Bolívia, conhecida no estado apenas de } \\
\text { duas localidades da Serra de Itatiaia, entre 2200-2400 m, (Brejo da Lapa e Prateleiras) apesar de se tratarem de } \\
\text { coleções recentes (Gradstein \& Costa 2003). }\end{array}$ \\
\hline $\begin{array}{l}\text { Diplasiolejeunea replicata (Spruce) } \\
\text { Steph. }\end{array}$ & VU & $\begin{array}{l}\text { Espécie disjunta entre o Norte dos Andes e o sudeste do Brasil (MG, RJ, SP, SC), conhecida no estado apenas } \\
\text { para Itatiaia (entre o Brejo da Lapa e Abrigo Rebouças) 2200-2400 m, apesar de se tratarem de coleções recentes } \\
\text { (Gradstein \& Costa 2003). }\end{array}$ \\
\hline $\begin{array}{l}\text { Lejeunea reflexistipula (Lehm. \& } \\
\text { Lindenb.) Gottsche }\end{array}$ & VU & $\begin{array}{l}\text { Espécie de distribuição neotropical, conhecida no Brasil para os estados de AM, PA, MT, ES, RJ, SP, sendo } \\
\text { recordada para o estado apenas pela coleção-tipo (Amphilejeunea reflexistipula (Lehm. \& Lindenb.) Gradst.), } \\
\text { da Serra da Estrela (Gottsche et al. 1844) realizada há mais de } 200 \text { anos. }\end{array}$ \\
\hline $\begin{array}{l}\text { Xylolejeunea crenata (Nees \& Mont.) } \\
\text { X.-L. He \& Grolle. }\end{array}$ & VU & $\begin{array}{l}\text { Espécie neotropical, que apesar de apresentar ampla distribuição no Brasil (RO, AM, PA, MA, PE, BA, RJ, } \\
\text { SP, MG), é conhecida para o estado por apenas uma coleção realizada por Regnell há mais de } 200 \text { anos sem } \\
\text { localidade (Gradstein \& Costa 2003). }\end{array}$ \\
\hline $\begin{array}{l}\text { Bazzania gracilis (Hampe \& } \\
\text { Gottsche) Steph. }\end{array}$ & VU & $\begin{array}{l}\text { Espécie neotropical, com ampla distribuição no Brasil (RR, AM, MG, RJ, SP), conhecida no estado apenas por } \\
\text { coleções antigas do município do Rio de Janeiro (Fulford 1963, Yano 1984) não tendo sido recoletada há mais } \\
\text { de } 100 \text { anos. }\end{array}$ \\
\hline Metzgeria psilocraspeda Schiffn. & VU & $\begin{array}{l}\text { Espécie endêmica do Brasil (MG, ES, RJ, SP, PR, SC, RS), considerada VU no país (Costa, dados não publicados). } \\
\text { No estado é conhecida apenas uma coleção para Itatiaia, Schiffner } 626 \text { (Costa, dados não publicados) não tendo } \\
\text { sido recoletada há mais de } 100 \text { anos. }\end{array}$ \\
\hline Metzgeria rufula Spruce & VU & $\begin{array}{l}\text { Espécie pantropical, ocorrendo no Brasil nos estados do RJ, SP, PR, considerada VU no país (Costa, dados não } \\
\text { publicados), e no estado conhecida apenas uma coleção antiga para o Corcovado (Stephani 1987) não tendo } \\
\text { sido recoletada há mais de } 100 \text { anos. }\end{array}$ \\
\hline Metzgeria subaneura Schiffn. & VU & $\begin{array}{l}\text { Espécie endêmica do Brasil (AC, MG, ES, RJ, SP, PR, RS), sendo considerada VU no país (Costa dados } \\
\text { não publicados). Para o estado é conhecida por apenas uma coleção recente para Itatiaia, Schäfer-Verwimp \& } \\
\text { Verwimp } 9242 \text { (RB) (Costa dados não publicados). }\end{array}$ \\
\hline Radula voluta Tayl. ex Gottsche et al. & VU & $\begin{array}{l}\text { Apesar de apresentar distribuição ampla no mundo e no Brasil (MG, ES, RJ, SP, SC, RS), é conhecida por } \\
\text { apenas uma coleção realizada há mais de } 100 \text { anos para o estado, para Itatiaia (Dusén 1903). }\end{array}$ \\
\hline
\end{tabular}

a categoria de vulnerável (VU). Os sete restantes permaneceram na mesma categoria $(\mathrm{EN})$.

Assim sendo, neste estudo são reconhecidas 75 espécies de hepáticas ameaçadas para o Rio de Janeiro, o que representa $21 \%$ da flora de hepáticas do estado, sendo duas criticamente em perigo (CR), oito em perigo (EN) e 65 vulneráveis (VU), além de quatro espécies que são consideradas quase ameaçadas (NT) e 12 com dados deficientes (DD).

A diferença observada entre a análise realizada anteriormente para o estado do Rio de Janeiro, aquelas realizadas para os demais estados do país, e os resultados aqui apresentados, reflete: 1) a importância do grau de conhecimento da brioflora do estado, que conta com checklist (Costa et al. 2005b), revisões (Costa, dados não publicados), floras e flórulas (Gradstein \& Costa 2003; Oliveira-e-Silva \& Yano 2000; etc.), análise da diversidade da brioflora (Costa et al. 2005a), e banco de dados de espécies (Costa et al. 2007); 2) os esforços de coleta realizados em áreas reconhecidas como lacunas no conhecimento da brioflora, tanto no que se refere a formações florestais (por exemplo, florestas submontanas), como áreas do estado (por exemplo, PE Desengano); e 3) o estudo realizado com as coleções depositadas em herbários (por exemplo, o herbário RB). Esse conjunto de fatores ex- plica o elevado número de espécies de hepáticas (75 táxons) aqui categorizadas como ameaçadas no estado do Rio de Janeiro. Além disso, vem de encontro às recomendações da IUCN quanto à realização de inventários florísticos, checklists, floras, revisões e análises regionais do status de conservação dos táxons.

A seguir são fornecidas informações para os dez táxons que mudaram de categoria de ameaça no estado.

Em perigo (EN) para criticamente em perigo (CR):

1. Bromeliophila natans (Steph.) R.M. Schust. - Espécie endêmica do sudeste do Brasil (Rio de Janeiro e São Paulo), ocorrendo na axila das folhas de bromélias (Vriesea glutinosa Lindl., Aechmea nudicaulis (L.) Griseb e Quesnelia arvensis (Vell.) Mez) em vegetação de restinga. Segundo Schuster (1994), a espécie está possivelmente extinta na localidade tipo (coletada por Ule em Mauá, próximo à cidade do Rio de Janeiro), sendo as únicas coleções recentes conhecidas, realizadas na Ilha do Cardoso, litoral do estado de São Paulo. Fato corroborado nos esforços de coleta realizados nas restingas do estado do Rio de Janeiro por Costa et al. (2006) e neste estudo, que não resultaram em novas coleções. Além disso, as regiões costeiras no estado 
Rio de Janeiro, como a praia de Mauá, são povoadas e sofrem forte pressão antrópica.

2. Southbya organensis Herzog - Espécie endêmica do estado do Rio de Janeiro, Morro Açu, PARNA-Serra dos Órgãos, município de Petrópolis, ocorrendo a ca. $2000 \mathrm{~m}$, e conhecida somente pela coleção tipo realizada por Luetzelburg (Herzog 1949). Os esforços de coleta realizados dentro dessa unidade de conservação, tanto na localidade tipo, quanto em outras áreas com a mesma altitude, entre os anos de 2003 e 2007, não resultaram em novas coleções.

Em perigo (EN) para vulnerável (VU):

1. Jensenia spinosa (Lindenb. \& Gottsche) Grolle-Espécie com distribuição afroamericana, que era conhecida no Brasil apenas por duas coleções antigas de Dusén, realizadas na Serra de Itatiaia, ca. 2200 m (Hässel de Menéndez 1961; Dusén 1903). Recentemente foi coletada no PARNA Itatiaia, nos campos de altitude, entre 2200-2400 m, e na floresta montana, ca. $750 \mathrm{~m}$ (Costa et al. 4442 RB; Santos et al. 681, 697 RB); e no PARNA Serra dos Órgãos, Pedra do Sino, ca. 2000 $\mathrm{m}$ (Costa et al. $4654 \mathrm{RB})$. Essas coleções aumentaram tanto o grau de proteção da espécie, quanto o número de localidades, variação altitudinal e extensão de ocorrência desta no estado.

2. Lejeunea grossiretis (Steph.) E. Reiner \& Goda Endêmica do Brasil (sul da Bahia e Rio de Janeiro), conhecida no estado do Rio de Janeiro até recentemente apenas pela coleção tipo, realizada por Rudolph em 1890, no município de Petrópolis, PARNA Serra dos Órgãos (Reiner-Drehwald \& Goda 2000). Recentemente foi coletada no PARNA Tijuca, floresta submontana, ca. 430 m (Santos et al. 524 RB); e no PE Desengano, floresta de terras baixas, ca. $50 \mathrm{~m}$ (Costa et al. 4831 $\mathrm{RB}$ ), aumentando o seu grau de proteção, sua variação altitudinal, número de localidades e extensão de ocorrência no estado.

Vulnerável (VU) para quase ameaçado (NT):

1. Calypogeia uncinulatula Herzog - As coleções brasileiras conhecidas haviam sido realizadas na década de 1920, nos estados de Minas Gerais e São Paulo (Bischler 1962, Fulford 1968). Entretanto, coletas recentes foram realizadas em duas localidades no estado do Rio de Janeiro, PARNA Serra dos Órgãos (Costa \& Coelho 4030 RB) e PARNA Itatiaia (Costa et al. 3800 RB), aumentando tanto a extensão de ocorrência, quanto o grau de proteção do táxon no estado, visto que estas unidades de conservação representam os maiores contínuos de Mata Atlântica no estado.

2. Cryptochila grandiflora (Lindenb. \& Gottsche) Grolle - Eram conhecidas apenas duas coleções para o Brasil, uma para o estado do Espírito Santo, PARNA Caparaó (Schäfer-Verwimp 1991) e outra para o estado do Rio de Janeiro, PARNA Itatiaia (Grolle 1971), ambas realizadas em formações alto-montanas, entre 1750-2500 $\mathrm{m}$. Recentemente foram realizadas quatro coleções em diferentes localidades situadas em UCs do estado do Rio de Janeiro, a saber: PE Três Picos, município de Nova Friburgo (Costa \& Gomes 984 RB); PARNA Itatiaia, Rio Campo Belo (Costa et al. $3820 \mathrm{RB}$ ), proximidades do Hotel Alsene (Costa et al. 4448 RB); e PARNA Serra dos Órgãos, Pedra do Papudo (Santos et al. 790, RB). Assim sendo, a extensão de ocorrência e o grau de proteção dessa espécie foram ampliados, embora esta continue restrita a localidades de altitudes elevadas no estado.

3. Heteroscyphus combinatus (Nees) Schiffn. - Conhecida para o estado do Rio de Janeiro apenas por duas coleções antigas, uma sem localidade e outra para a Serra dos Órgãos (Fulford 1976), recentemente foi coletada em diferentes localidades e UCs do estado: município de Nova Friburgo (Costa 525, RB), PE Desengano (Santos et al. 856, RB) e RPPN El Nagual (Santos et al. 85 p.p., RB). Todas as coleções foram realizadas sobre rocha na margem de riachos. Essa restrição de habitat, possivelmente torna a espécie subamostrada no estado.

4. Lophocolea mandonii Steph. - Espécie com distribuição disjunta entre a Bolívia e o sudeste do Brasil (MG, RJ), conhecida no estado do Rio de Janeiro somente para o PARNA Itatiaia (Fulford 1976, Gradstein \& Costa 2003). Recentemente teve sua extensão de ocorrência ampliada com as novas coleções realizadas em diferentes UCs do estado: PARNA Tijuca (Santos et al. 521 p.p. RB) e RPPN El Nagual (Santos \& Costa 336, 351, $353 \mathrm{RB})$.

Vulnerável (VU) para não ameaçada (LC)

1. Plagiochila patula (Sw.) Lindenb. - Espécie de distribuição Neotropical, ocorrendo no Brasil em diferentes regiões e estados (AC, BA, MG, RJ e SP). As coletas recentes realizadas no estado do Rio de Janeiro aumentaram tanto a extensão de ocorrência, quanto o grau de proteção dessa espécie, sendo encontrada em localidades situadas em UCs que formam um contínuo de Mata Atlântica, a saber: PARNA Itatiaia (Costa \& Gradstein 3732 RB); PARNA Serra dos Órgãos (Santos et al. 773 RB); PE Desengano (Santos et al. 879 RB); REBIO Tinguá, município Nova Iguaçu (Costa et al. 4053 RB); e RPPN El Nagual (Santos et al. 75, 232 RB).

O estado do Rio de Janeiro é considerado um dos centros de diversidade e endemismo do Brasil (Guedes-Bruni \& Lima 1997; Rocha et al. 2003; Gradstein \& Costa 2003; entre outros), contando com um elevado número de táxons endêmicos do país ou com distribuição predominando na região sudeste. Isso, em parte, explica os resultados aqui encontrados na análise dos padrões de distribuição dos 75 táxons considerados com algum grau de ameaça no estado (Tab. 1), onde predominaram espécies neotropicais (26 espécies - 35\%) seguidas daquelas com distribuição disjunta (25 espécies - 33\%) e endêmica do Brasil (15 espécies - 20\%). 
Dentre as espécies neotropicais, 16 (61\%) ocorrem apenas na região sudeste no Brasil.

As espécies disjuntas geralmente apresentam restrição de habitat no estado, como por exemplo, campos de altitude, e por isso possuem maior grau de ameaça, onde se destacam sete espécies com distribuição disjunta com os Andes e que no estado estão presentes somente nas formações altomontanas dos PARNAs Itatiaia e Serra dos Órgãos. Safford (2007) comenta que os campos de altitude representam um foco de "vegetação andina" no Brasil, e que as mudanças climáticas podem levar ao desaparecimento desse importante ecossistema num futuro próximo.

Em relação à distribuição dos 75 táxons ameaçados dentro do estado do Rio de Janeiro (Fig. 1), percebe-se que a maioria (51 espécies - 68\%) ocorre no PARNA Itatiaia, dos quais 38 (51\%) são encontrados exclusivamente nessa unidade de conservação. Esses resultados corroboram a importância do PARNA Itatiaia para a conservação da diversidade de briófitas no estado, bem como de espécies ameaçadas ou endêmicas, como por exemplo, Colura itatyana Steph. Outras áreas e unidades de conservação do estado que se destacam em termos de número de espécies ameaçadas são o PARNA Serra dos Órgãos (17 espécies) e os municípios do Rio de Janeiro (12 espécies) e de Petrópolis (seis espécies).

Analisando a distribuição dos táxons ameaçados nos diferentes cinturões altitudinais da Mata Atlântica do estado (Fig. 2), verifica-se que eles predominam nas formações alto-montana (>1500 m), com 45 táxons, e montana (500$1500 \mathrm{~m}$ ), com 37 táxons; destacando a importância da conservação dessas áreas. Essas formações são também aquelas que abrigam a maior riqueza e o maior número de táxons exclusivos de hepáticas no estado do Rio de Janeiro (Santos \& Costa, inédito).

No que se refere ao número de espécies ameaçadas por família, verificou-se que, das 31 famílias de hepáticas ocorrentes no estado, somente 16 apresentam espécies ameaçadas (Fig. 3). Dessas, três famílias concentram 52\% das espécies, destacando-se Lejeuneaceae, com 23 espécies (16\% do total de espécies da família que ocorre no estado); Jungermanniaceae, com oito espécies (57\%); e Lepidoziaceae, com seis espécies (27\%). A família Arnelliaceae apresenta somente dois gêneros e duas espécies no país, sendo essas duas espécies categorizadas como ameaçadas no estado, Gongylanthus liebmanianus (Lindenb. \& Gottsche) Steph. (VU) e Southbya organensis (CR).

\section{Principais ameaças à flora de hepáticas do estado do Rio de Janeiro}

Segundo Hallinbäck \& Hodgetts (2000), a perda e degradação de habitat são as mais sérias ameaças às briófitas em todo o mundo. A degradação reduz a qualidade do habitat, causando o desaparecimento das espécies sensíveis, já a fragmentação conduz ao isolamento das comunidades de briófitas, visto que a dispersão e a reprodução são diretamente afetadas. Além disso, a fragmentação e destruição do habitat representam as maiores ameaças para espécies com distribuição restrita ou endêmica.

O estado do Rio de Janeiro apresentava 97\% de sua área recoberta pela Mata Atlântica. Atualmente, a cobertura vegetal remanescente encontra-se reduzida a ca. 19\% de sua área original (Fundação S.O.S. Mata Atlântica 2002), e o resultado do contínuo processo de remoção das áreas florestadas é a formação de fragmentos de diferentes tamanhos, causando a insularização de grande parte .das populações animais e vegetais (Rocha et al. 2003).

A área protegida por unidades de conservação no estado do Rio de Janeiro é de ca. 13\% da sua área total, e a maioria dessas unidades apresenta formações de floresta atlântica montana e alto-montana (SEMADS 2001).

Rocha et al. (2003) destacam que, apesar dos remanescentes florestais do Rio de Janeiro estarem relativamente protegidos por unidades de conservação, ainda estão sob forte pressão de degradação em seu entorno e área interna, devido ao desmatamento para pastagens e culturas, urbanização, favelização, fiscalização insuficiente das UCs, comércio ilegal de espécies da fauna e flora, retirada de madeira e introdução de espécies exóticas, o que eviden-

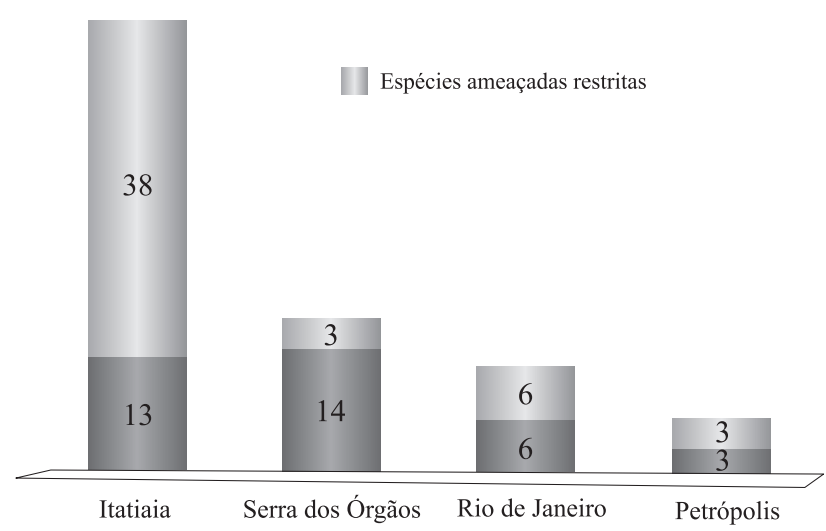

Figura 1. Principais áreas do Estado do Rio de Janeiro, Brasil, em termos de número de espécies ameaçadas, destacando em cinza claro os táxons restritos a essas áreas.

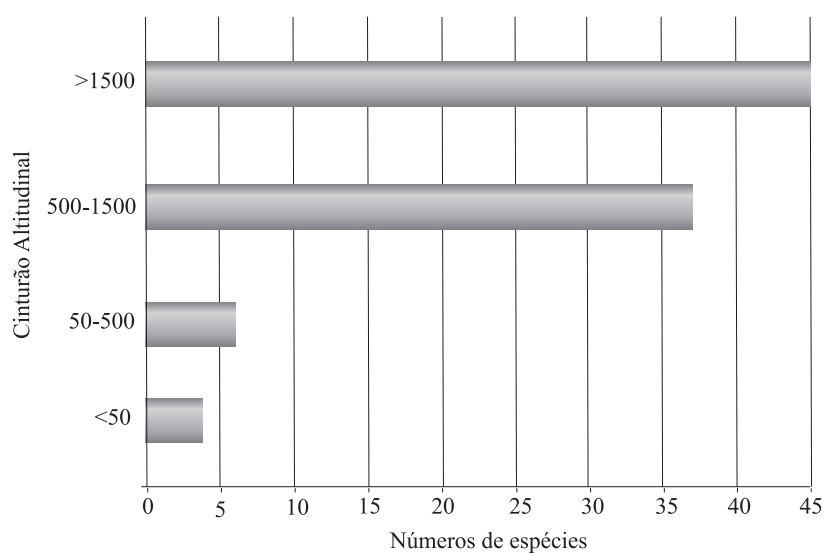

Figura 2. Número de espécies de hepáticas ameaçadas por cinturão altitudinal da Mata Atlântica do Estado do Rio de Janeiro, Brasil. 


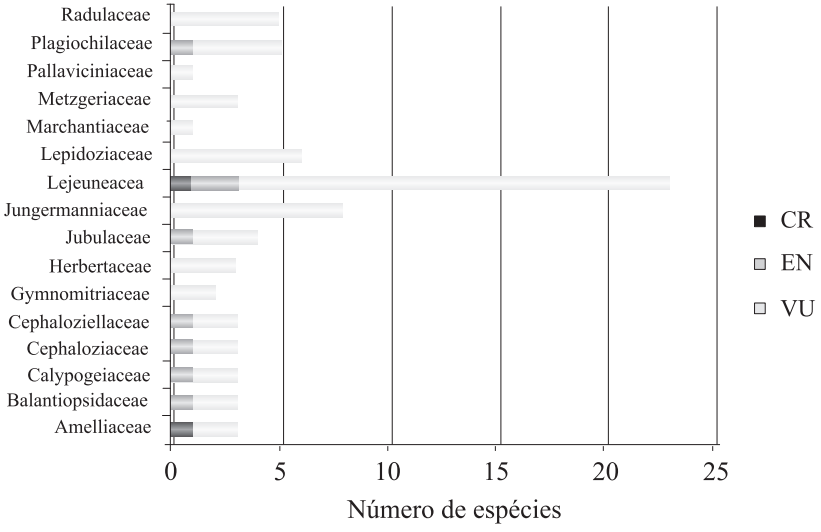

Figura 3. Número de espécies de hepáticas ameaçadas do Estado do Rio de Janeiro, Brasil, por família.

cia a necessidade de proteção efetiva dessas unidades de conservação.

Como exemplos de unidades ou áreas no estado que sofrem com a degradação e que contam com um grande número de espécies de hepáticas ameaçadas, temos:

1. PARNA Itatiaia - concentra uma alta diversidade de espécies, alto número de táxons de hepáticas endêmicos e ameaçados no estado, e onde a questão fundiária (propriedades privadas no interior do parque), pastagem e culturas mistas cíclicas e permanentes ainda constituem as maiores ameaças (Rocha et al. 2003).

2. PARNA Serra dos Órgãos - a segunda unidade em importância para a flora de hepáticas do estado, onde as maiores ameaças são a presença da BR-116 cortando o parque, projetos de instalação de empreendimentos incompatíveis (gasodutos, estações de rádio e de telefonia móvel, etc.), moradias e produção rural em áreas não regularizadas, extração ilegal de palmito e de plantas ornamentais, caça comercial e esportiva de animais silvestres e visitação predatória (IBAMA 2007).

3. Município do Rio de Janeiro - a terceira área em importância no estado, e cujos remanescentes florestais vêm sofrendo intensa degradação devido ao crescimento urbano (favelas e empreendimentos imobiliários), espécies introduzidas (capinzais, jaqueirais, etc.), monocultura de banana, etc. (Rocha et al. 2003).

\section{Recomendações}

Gradstein \& Raeymaerkers (2000), no capítulo Panorama Regional (América Tropical) do Plano de Ação para as Briófitas, indicam quatro recomendações para a conservação da brioflora: reconhecimento de cinco áreas críticas; reconhecimento dos centros de diversidade e endemismo; proteção de habitats para a sobrevivência das espécies e comunidades de briófitas; e formulação de programas multidisciplinares para promover a conservação.

Dessas recomendações, três são aqui indicadas para a conservação da flora de hepáticas do estado do Rio de Janeiro, a saber:

1) Reconhecer a Mata Atlântica do estado como área crítica para conservação.
2) Proteger habitats para assegurar a sobrevivência das espécies e comunidades de briófitas, como pequenas UCs ou grandes áreas florestais. Como um exemplo de pequenas UCs que servem para proteção das espécies fornecendo e mantendo microhabitats de forma adequada, temos a RPPN El Nagual (Santos \& Costa 2008). Para Gradstein \& Raeymaerkers (2000), entretanto, grandes áreas florestais que incluam gradientes e fontes de reposição de forófitos, são fundamentais para a conservação de espécies de hepáticas florestais. Assim sendo, no estado do Rio de Janeiro a regulamentação da questão fundiária do PARNA Itatiaia é fundamental para a conservação da brioflora em uma das maiores áreas de contínuo de Mata Atlântica no estado.

3) Estimular inventários através do treinamento de estudantes em diferentes níveis; elaborar floras; incrementar os herbários com coleções de briófitas; divulgar o grupo vegetal para o público em geral (manuais, sites, vídeos, etc.); preparar e divulgar listas vermelhas; identificar os centros de diversidade; realizar estudos ecológicos; monitorar as espécies ameaçadas; integrar a conservação das briófitas em outros esforços de conservação.

Em relação às recomendações da IUCN, diversas ações de conservação vêm sendo desenvolvidas com a brioflora do estado do Rio de Janeiro a partir do ano de 2003, com a implantação do "Projeto Flora do estado do Rio de Janeiro: briófitas", a saber: elaboração de banco de dados de espécies (Costa et al. 2007); publicação de checklist e de um diagnóstico da brioflora apresentando a lista vermelha para o estado (Costa et al. 2005a,b); realização de inventários em áreas ou formações vegetacionais consideradas lacunas no conhecimento (PE Desengano, RPPN El Nagual, PARNA Tijuca, etc.); elaboração de um Manual de Briologia direcionado a estudantes de graduação (Costa et al., inédito); criação de uma home-page com informações gerais, projetos e banco de dados da brioflora (www.jbrj.gov.br/pesquisa/ div_tax/briofitas); elaboração de flórulas e análises florísticas (Costa \& Lima 2005; Costa et al. 2006b; Vaz-Imbassahy \& Costa 2006a,b; Santos \& Costa 2008); e estabelecimento das prioridades para a conservação da brioflora no que se refere a centros de diversidade e endemismo, onde estão as espécies ameaçadas ou vulneráveis UCs e localidades cuja brioflora é desconhecida, e criação e/ou ampliação de UCs (Costa \& Farias, 2008).

\section{Agradecimentos}

Nós gostaríamos de agradecer à Fundação O Boticário de Proteção à Natureza (projeto 0709_20061) e ao Instituto de Pesquisas Jardim Botânico do Rio de Janeiro, pelo financiamento da pesquisa. E à Coordenação de Aperfeiçoamento de Pessoal de Nível Superior (CAPES), pela bolsa de mestrado concedida à segunda autora.

\section{Referências bibliográficas}

Bescherelle, E. 1893. Liste des hépatiques récoltées aux environs de Rio de Janeiro (Brésil) par M. Glaziou, et déterminées par M. Stephani. Revue Bryologique 20: 59-60. 
Bischler, H. 1962. The genus Calypogeia Raddi in Central and South America. I. Introduction and subgenera Mnioloma and Caracoma. Candollea 18: 19-51.

Costa, D.P. 1992. Hepáticas do Pico da Caledônea. Nova Friburgo, Rio de Janeiro, Brasil. Acta Botanica Brasilica 6: 3-39.

Costa, D.P. 1997. Bryopyta e Hepatophyta. Pp. 37-43. In: M.C.M. Marques (ed.). Mapeamento da cobertura vegetal e listagem das espécies ocorrentes na APA Cairuçu, Parati, RJ. Série Estudos e Contribuições 13. Rio de Janeiro, Jardim Botânico do Rio de Janeiro.

Costa, D.P. 1999. Epiphytic bryophyte diversity in primary and secondary lowland rainforests in southeastern Brazil. The Bryologist 102: 320-326.

Costa, D.P. 2008. Metzgeriaceae. Flora Neotropica. Monograph 102. New York, The New York Botanical Garden.

Costa, D.P. Metzgeriaceae (Metzgeriales, Hepatophyta) no Brasil. Tese de doutorado em Ciências Biológicas (Botânica), Universidade de São Paulo, Instituto de Biociências, São Paulo (dados não publicados).

Costa, D.P. \& Farias, C.P. 2008. Conservation priorities for the bryoflora of Rio de Janeiro state, Brazil. Journal of Bryology 30: 133-142.

Costa, D.P. \& O. Yano. 1988. Hepáticas talosas do Parque Nacional da Tijuca, Rio de Janeiro, Brasil. Acta Botanica Brasilica 1 (Supl.): 73-82.

Costa, D.P. \& O. Yano. 1998. Briófitas da restinga de Macaé, Rio de Janeiro, Brasil. Hoehnea 25: 99-119.

Costa, D.P. \& Lima, F.M. 2005. Moss diversity in the tropical rainforest of Rio de Janeiro, Southeastern Brazil. Revista Brasileira de Botânica 28(4): 671-685.

Costa, D.P. Imbassahy, C.A.A. \& Silva, V.P.A.V. 2005a. Diversidade e importância das espécies de briófitas na conservação dos ecossistemas do estado do Rio de Janeiro. Rodriguésia 56(87): 13-49.

Costa, D.P. Imbassahy, C.A.A. \& Silva, V.P.A.V. 2005b. Checklist and distribution of mosses, liverworts and hornworts of Rio de Janeiro state, Brazil. The Journal of the Hattori Botanical Laboratory 98: $259-298$

Costa, D.P. Pôrto, K.C., Starling, F., Santos, N.D. \& Yano, O. 2006a. Briófitas. Pp. 42-43. In: G.M. Drummond, C.S. Martins \& M.P. Mendonça (eds.). Revisão das listas das species da flora e fauna ameaçadas de extinção do estado de Minas Gerais. Relatório Final. Belo Horizonte, Fundação Biodiversitas.

Costa, D.P. Imbassahy, C.A.A., Almeida, J.S.S., Santos, N.D., VazImbassahy, T.F. 2006b. Diversidade das briófitas nas restingas do estado do Rio de Janeiro. Boletim do Instituto de Botânica 18: 131-140.

Costa, D.P. Imbassahy, C.A.A. \& Silva, V.P.A.V. \& Monteiro, M.D. 2007. Banco de Dados - Briófitas do estado do Rio de Janeiro. http:// www.jbrj.gov.br/pesquisa/div tax/briofitas (acessado em 10/12/2007).

Costa, D.P. \& Farias, C.P. Conservation priorities for the bryoflora of Rio de Janeiro state, Brazil. Journal of Bryology (submetido em 2007).

Costa, D.P. Almeida, J.S.S., Santos, N.D., Gradstein, S.R. \& Churchill, S.P. Manual de Briologia (inédito)

Dusén, P. 1903. Sur la flore de la Serra do Itatiaya au Brésil. Arquivos do Museu Nacional do Rio de Janeiro 13: 1-119.

Fulford, M.H. 1963. Manual of the leafy Hepaticae of Latin America I. Memoirs of The New York Botanical Garden 11: 1-172.

Fulford, M.H. 1968. Manual of the leafy Hepaticae of Latin America III. Memoirs of The New York Botanical Garden 11: 277-392.

Fulford, M.H. 1976. Manual of the leafy Hepaticae of Latin America IV. Memoirs of The New York Botanical Garden 11: 393-535.

Fundação CIDE 2006. O Rio de Janeiro em dados. Fundação CIDE, Centro de Informações e Dados do Rio de Janeiro, Governo do Estado do Rio de Janeiro, Rio de Janeiro.

Fundação SOS Mata Atlântica. 2002. Atlas da evolução dos Remanescentes Florestais da Mata Atlântica, período 1995-2000: relatório final. São Paulo, Fundação S.O.S. Mata Attântica/INPE.

Galindo-Leal,C. \& Câmara, I.G. 2005. Status do hotspot Mata Atlântica: uma síntese. Pp. 3-12. In: C. Galindo-Leal \& I.G. Câmara (eds.). Mata Atlântica: biodiversidade, ameaças e perspectivas. São Paulo: Fundação SOS Mata Atlântica - Belo Horizonte: Conservação Internacional.

Gottsche, C.M., Lindengerg, J.B.G. \& Nees von Esenbeck,C.G. 1844. Synopsis Hepaticarum i-xxiv+1-835. J-A. Meissner ed., Hamburg.

Gradstein, S.R. \& Costa, D.P. 2003. The Hepaticae and Anthocerotae of Brazil. Memoirs of The New York Botanical Garden 87: 1-336.
Gradstein, S.R. \& Raeymaekers, G. 2000. Regional overviews. Tropical America (incl. Mexico). Pp. 38-44. In: T. Hallinbäck \& N. Hodgetts (eds.) Mosses, liverworts and hornworts. Status Survey and Conservation Action Plan for Bryophytes. IUCNSSC Bryophyte Specialist Group. IUCN, Gland, Switzerland and Cambridge, UK.

Grolle, R. 1971. Jamesoniella und Verwandt. Feddes Repertorium 82: 1-100

Guedes-Bruni, R.R. \& Lima, H.C. 1997. Mountain Ranges of Rio de Janeiro, South-eastern Brazil. Pp. 376-380. In: S.D. Davis, V.H. Heywood, O. Herrera-MacBryde, J. Villa-Lobos \& A.C. Hamilton (eds.). Centres of plant diversity. A guide and strategy for their conservation. Vol. 3. The Americas. WWF, IUCN.

Hallingbäck, T. \& Hodgetts, N. 2000. Mosses, liverworts \& hornworts: a status survey and conservation action plan for bryophytes. IUCN, Gland.

Hallingbäck, T., Hodgetts, N., Raeymaekers, G., Schumacker, R., Sérgio, C., Söderström, L., Stewart, N. \& Vána, J. 1998. Guidelines for application of the revised IUCN threat categories to bryophytes. Lindbergia 23: 6-12.

Hässel de Menéndez, G.G. 1961. Las espécies Sudamericanas del género Pallavicinia. Boletin de la Sociedade Argentina de Botanica 9: 261282.

Hatcher, R.E. 1961. A monograph of the genus Isotachis (Hepaticae). Separately printed from Nova Hedwigia II, 4 and III, I.

Herzog, T. 1949. Miscellania Bryologica. I. Neotropica. Memoranda Societalis pro Fauna Flora Fennica 25:43-73.

Hooker, J.D. \& Wilson, W. 1844. Enumeration of the mosses and hepatics collected in Brazil by George Gardner. London Journal of Botany 3: $149-167$.

IBAMA. 2007. Parque Nacional da Serra dos Órgãos. Plano de Manejo. Documento Síntese. Disponível em www.parnaso.com.br, acessado em 15/11/2007.

IUCN. 2001. IUCN Red List Categories and Criteria: Version 3.1. IUCN Species Survival Commission. IUCN, Gland, Switzerland and Cambridge, UK. ii $+30 \mathrm{pp}$.

IUCN. 2003. Guidelines for Application of IUCN Red List Criteria at Regional Levels: Version 3.0. IUCN Species Survival Commission. IUCN, Gland, Switzerland and Cambridge, UK. ii + 26 pp.

Massalongo, C. 1911. Liste des Hépatiques du Brésil recueillies par Mons. V. Mazzucchelli (1904). Revue Bryologique 38: 9-12.

Mittermeier, R.A., Gil, P.R. Hoffmann, M., Pilgrim, J., Brooks, T., Mittermeier, C.G., Lamourex, J. \& Fonseca, G.A.B. 2004. Hotspots Revisited. Earth's Biologically Richest and Most Endangered Terrestrial Ecorregions. Cemex, Washington, DC.

Molinaro, L.C. \& Costa, D.P. 2001. Briófitas do arboreto do Jardim Botânico do Rio de Janeiro. Rodriguésia 52: 107-124.

Montagne, C. 1839. Cryptogamae brasiliensis seu plantae cellulares quas in itinere per Brasilian a celeb. Auguste de Saint-Hilaire collectas recensuit observatoribusque nonnulis illustravit. Annales des Sciences Naturelles: Botanique 2, 12: 42-55.

Myers, N., Mittermeier, R.A., Mittermeier, C.G., Fonseca, G.A.B., Kent, J. 2000 Biodiversity hotspots for conservation priorities. Nature 403: 853-858.

Nees ab Esenbeck, C.G. 1833. Hepaticae. Pp: 293-390. In: C.F.P. Martius. Flora Brasiliensis. Stuttgartiae et Turbingae. J.G. Cottal ed. 1.

Oliveira e Silva, M.I.M.N. \& Yano, O. 2000. Anthocerotophyta e Hepatophyta de Mangaratiba e Angra dos Reis, Rio de Janeiro, Brasil. Boletim do Instituto de Botânica 13: 1-102.

Oliveira e Silva, M.I.M.N. Milanez, A.I. \& Yano, O. 2002. Aspectos ecológicos de briófitas em áreas preservadas de mata atlântica, Rio de Janeiro, Brasil. Tropical Bryology 22: 77-102

Pinto, L.P., Bedê, L., Paese, A., Fonseca, M. Paglia, A. \& Lamas, I. 2006. Mata Atlântica Brasileira: os desafios para conservação da biodiversidade de um hotspot mundial. Pp. 91-118. In: C.F.D. Rocha, H.G. Bergallo, M.V. Sluys, \& M.A.S. Alves (eds.). Biologia da Conservação: essências. São Carlos, RiMa.

Pôrto, K.C. \& Germano, S.R. 2002. Biodiversidade e importância das briófitas na conservação dos ecossistemas naturais de Pernambuco. Pp. 125-152. In: M. Tabarelli \& J.M.C. Silva. Diagnóstico da biodiversidade de Pernambuco. Recife, Massangana.

Projeto Flora do Estado do Rio de Janeiro: Bases para o Uso Sustentável da Diversidade Vegetal. (2002) (inédito). 
Raddi, G. 1822. Crittogame brasiliane racclote e descritte (preprint) (Também publicado em: 1823. Memorie di matematica e di fisica della società italiana delle scienze residente in Modena 19: 27-57.

Rambaldi, D. M., Magnani, A., Ilha, A., Lardosa, E., Figueiredo, P., Oliveira, R. F. 2003. A Reserva da Biosfera da Mata Atlântica no Estado do Rio de Janeiro. Série Estados e Regióes da RBMA. Caderno da Reserva da Biosfera da Mata Atlântica. Rio de Janeiro: CNRBMA.

Reiner-Drehwald, M.E. \& Goda, A. 2000. Revision of the genus Crossotolejeunea (Lejeuneaceae, Hepaticae). The Journal of the Hattori Botanical Laboratory 89: 1-54.

Rocha, C.F.D., Bergallo, H.G., Alves, M.A.S. \& Sluys, M.V. 2003. A biodiversidade nos grandes remanescentes de florestais do estado do Rio de Janeiro e nas restingas da Mata Atlântica. São Carlos, RiMa.

Safford, H.D. 2007. Brazilian Páramos IV. Phytogeography. Journal of Biogeography 34:1701-1722.

Santos, N.D. \& Costa, D.P. 2008. A importância de Reservas Particulares do Patrimônio Natural para a conservação da brioflora da Mata Atlântica no estado do Rio de Janeiro: um estudo em El Nagual, Magé. Acta Botanica Brasilica 22: 359-372.

Santos, N. D. \& Costa, D.P. 2010. Altitudinal zonation of liverworts in the Atlantic Forest, Southeastern Brazil. The Bryologist (no prelo).

Santos, N.D. \& Costa, D.P. Diversidade de hepáticas na Mata Atlântica do estado do Rio de Janeiro, Brasil (inédito).

Schäfer-Verwimp, A. 1991. Contribution to the knowledge of the bryophyte flora of Espírito Santo, Brazil. The Journal of the Hattori Botanical Laboratory 69: 147-170.

Schuster, R.M. 1994. Studies on Lejeuneaceae, I. Preliminary studies on new genera of Lejeuneaceae. The Journal of the Hattori Botanical Laboratory 75: 211-235.

SEMADS. 2001. Atlas das Unidades de Conservação do Estado do Rio de Janeiro. São Paulo, Metalivros.

Silva, J.M.C. \& Casteleti, C.H.M. 2005. Estado da biodiversidade da Mata Atlântica brasileira. Pp. 43-60. In: C. Galindo-Leal, \& I.G. Câmara (eds.). Mata Atlântica: biodiversidade, ameaças e perspectivas. São Paulo, Fundação SOS Mata Atlântica, Belo Horizonte, Conservação Internacional.

Spruce, R.M. 1888. Hepaticae in prov. Rio de Janeiro a Glaziou lectae, a R. Spruce determinatae. Revue Bryologique 15(3): 33-34.

Stephani, F. 1897. Die Lebermoose der ersten Regnell'schen Expedition nach Südamerika. Bihang til Kongliga Svenska VetenskapsAkademiens Handlingar 23: 1-36.

Vaz-Imbassahy, T.F. \& Costa, D.P. 2006a. Os gêneros Brymela, Callicotella, Crossomitrium, Cyclodictyon, Hookeriopsis e Hypnella no estado do Rio de Janeiro. Acta Botanica Brasilica 20: 955-973.

Vaz-Imbassahy, T.F. \& Costa, D.P. 2006b. Os gêneros Lepidopilidium, Lepidopilum, Pilotrichum e Thamniopsis no estado do Rio de Janeiro. Acta Botanica Brasilica 20: 975-993.

Yano, O. 1984. Checklist of Brazilian liverworts and hornworts. The Journal of the Hattori Botanical Laboratory 56: 481-548.

Yano, O. 1989. An additional checklist of Brazilian bryophytes. The Journal of the Hattori Botanical Laboratory 66: 371-434.

Yano, O. 1995. A new additional annotated checklist of Brazilian bryophytes. The Journal of the Hattori Botanical Laboratory 78: 137-182.

Yano, O. 1996. A checklist of Brazilian bryophytes. Boletim do Instituto de Botânica 10: 47-232.

Yano, O. 2006. Novas adições ao catálogo de briófitas brasileiras. Boletim do Instituto de Botânica 17: 1-142.

Yano, O. \& Costa, D.P. 1998. Briófitas da restinga de Massambaba, Rio de Janeiro. Pp. 144-152. In: Anais do III Simpósio de Ecossistemas da Costa Brasileira. São Paulo, ACIESP.

Yano, O. \& Peralta, D.F. 2007. As briófitas ameaçadas de extinção no Espírito Santo. Pp. 81-87. In: M. Simonelli \& C.N. Fraga. Espécies da Flora Ameaçadas de Extinção no Estado do Espírito Santo. Vitória, Ipema.

Yano, O. Costa, D.P. Pôrto, K.C. \& Peralta, D.F. 2005. Lista de espécies da flora brasileira ameaçada de extinção: briófitas. http://www.biodiversitas.org.br/floraBr/grupo3fim.asp (acesso em 13/02/2008). 